determined at the mRNA and protein levels. Finally, expression of LOXL2 was determined in an Aspergillus fumigatus model of asthma.Asthmatic HASM cells activated 3-fold higher levels of TGF $\beta$ basally than non-asthmatic cells $(p<0.01)$. Both diseased and control HASM cells increased TGF $\beta$ activation in response to methacholine confirming our previous data (Tatler et al 2011). A collagen gel contraction assay demonstrated that asthmatic HASM cells were hypercontractile compared with non-asthmatic cells under basal conditions $(p<0.05)$ and that contractility weakly correlated with amount of TGF $\beta$ activated $(p<0.05)$. Importantly, culturing non-asthmatic HASM cells on asthmatic ECM led to increased TGF $\beta$ activation $(\mathrm{p}<0.05)$ and culturing asthmatic HASM cells on non-asthmatic ECM decreased TGF $\beta$ activation $(\mathrm{p}<0.05)$. mRNA Expression of the ECM crosslinking enzymes LOXL2 and LOXL3 was significantly increased in asthmatic HASMs $(\mathrm{p}<0.05)$. Finally, LOXL2 protein was increased in asthmatic HASMs cells, and increased in the airway smooth muscle layer of animals challenged repeatedly with Asp. $f$ compared with control challenged animals. In conclusion HASM cells derived from asthmatic patients exhibit enhanced activation of TGF $\beta$ compared with non-asthmatic HASM cells. This may be driven by the diseased ECM since asthmatic HASMs cells exhibit aberrant expression of ECM crosslinking enzymes.

\section{S87 REGULATION OF TYPE 2 CYTOKINE RELEASE BY EPITHELIAL CELLS: CHARACTERISATION OF SOLUBLE FACTOR PATHWAYS AND CHARACTERISATION OF POTENTIAL MEDIATORS}

A Southern, S Layzell, JL Cane, TJ Powell, ID Pavord. Respiratory Medicine Unit and NIHR Oxford Biomedical Research Centre Respiratory Theme, Nuffield Department of Clinical Medicine, University of Oxford, Oxford, UK

\subsection{6/thoraxjnl-2017-210983.93}

Introduction Type 2 cytokines such as IL-13, IL-4 and IL-5 have been shown to play important roles in the pathogenesis of asthma. One source of these cytokines is type 2 CD4 +or CD8+T cells. We have shown that epithelial cells have inhibitory effects on these $\mathrm{T}$ cells and that this regulatory effect could be defective in asthma. We have previously shown that type $2 \mathrm{~T}$ cell lines release less IL-13 in the presence of epithelial cells and others have shown that epithelial cells are able to reduce division of CD4 $+\mathrm{T}$ cells. We wished to extend these studies to determine whether bulk cultures of PBMC were able to release IL-13 and whether this IL-13 was regulated by epithelial cells and whether this was mediated by direct cell contact.

Methods We used PBMC from healthy donors and cultured cells in the presence and absence of epithelial cells with titrated doses of IL-2. We used transwells and epithelial cell supernatants to determine whether supernatants were also able to reduce type 2 cytokine secretion. We used size exclusion centrifugation to split supernatants into different fractions.

Results After culture of PBMC for 5 days in IL-2, IL-13 release (pg/10 ${ }^{6}$ cells+/-SD) was $509.95+/-84.95$ and was reduced to $37.3+/-7.4$ by A549 epithelial cells separated by a transwell. Titration of A549 cells established that inhibition was cell number dependent. Inhibition was not due to scavenging of IL-13 by epithelial cells during co-culture. Less IL-
13 was secreted by IL-2 treated PBMC (pg/10 ${ }^{6}$ cells +/-SD) in the presence of $50 \% \mathrm{v} / \mathrm{v}$ supernatant from healthy HBEC 49 $+/-7$ or asthma HBEC 90+/-21 $\mathrm{p}=0.0023$. IL-5: HHBEC 14 $+/-5$ AHBEC $26+/-12$ not significant. Splitting the HBEC supernatant into different size fractions showed that the fraction over $3 \mathrm{kD}$ was less inhibitory than the fraction under 3 $\mathrm{kD}$.

Conclusion There may be a soluble mediator secreted by epithelial cells that is less than $3 \mathrm{kDa}$ in mass that is able to inhibit type 2 cytokine release from PBMC. These inhibitory factor(s) could contribute to the regulation of type 2 cells that and affect asthma pathogenesis.

\section{S88 MICRORNAS REGULATE GENOME-WIDE TRANSLATION IN SEVERE ASTHMA BRONCHIAL EPITHELIAL CELLS AS REVEALED BY FRAC-SEQ}

${ }^{1} \mathrm{RT}$ Martinez-Nunez, ${ }^{2} \mathrm{H}$ Rupani, ${ }^{3} \mathrm{M}$ Niranjan, ${ }^{4} \mathrm{PH}$ Howarth, ${ }^{2} \mathrm{~T}$ Sanchez-Elsner. ${ }^{7}$ King's College London, London, UK; ${ }^{2}$ Clinical and Experimental Sciences, Faculty of Medicine, University of Southampton, Southampton, UK; ${ }^{3}$ School of Electronics and Computer Science, University of Southampton, Southampton, UK; ${ }^{4}$ Southampton NIHR Respiratory Biomedical Research Unit, University Hospital Southampton NHS Foundation Trust, Southampton, UK

10.1136/thoraxjnl-2017-210983.94

Severe asthma represents a significant unmet clinical need and the molecular basis for disease persistence remains inadequately understood. Bronchial epithelial cells, at the interface of environment/tissue, are central to asthma pathogenesis. There is thus a need to evaluate genome-wide changes between health and asthma to better understand the molecular mechanisms underlying disease. The vast majority of genomewide measurements have focused on determining changes at the DNA or mRNA levels, with little attention paid to how and which mRNAs are actually translated into protein. This may not disclose changes happening at the protein level, since mRNA and protein expression correlate poorly. To determine translation and its regulation in bronchial epithelial cells in severe asthma patients we analysed paired genome-wide expression of transcriptional (cytoplasmic) and translational (polyribosome-bound) mRNA levels employing Frac-seq (subcellular fractionation and RNA-sequencing) in primary bronchoepithelium in health and severe asthma patients. We also integrated those data with genome-wide profiling of microRNAs to understand their role in gene expression and impact on the pathophysiology of severe asthma bronchial epithelium. We found both genes (=all isoforms of a gene) and mRNA isoforms differentially expressed in severe asthma airways cells, with dysregulated transcriptional mRNA levels (194 genes) showing little overlap with dysregulated translational mRNA (243 genes) expression. We determined novel inflammatory and remodelling pathophysiological mechanisms disclosed solely by polyribosome-bound mRNAs, centred in epithelium remodelling and repair pathways. We also reveal six dysregulated microRNAs accounting for $\sim 90 \%$ of all cellular microRNA targeting, displaying preferential targeting of $\sim 50 \%$ of mRNAs undergoing translation in severe asthma airways cells. Thus, microRNAs in human severe asthma are major regulators of translation in airways epithelium and offer potential as future therapeutic targets. 